ARTICLE

\title{
The L-type calcium channel blocker, isradipine, attenuates cue- induced cocaine-seeking by enhancing dopaminergic activity in the ventral tegmental area to nucleus accumbens pathway
}

\author{
Nii A. Addy ${ }^{1,2,3}$, Eric J. Nunes ${ }^{1}$, Shannon M. Hughley ${ }^{1}$, Keri M. Small ${ }^{1}$, Sarah J. Baracz ${ }^{1}$, Joshua L. Haight ${ }^{1}$ and Anjali M. Rajadhyaksha ${ }^{4,5}$
}

Previous preclinical and clinical investigations have focused on the L-type calcium channel (LTCC) as a potential therapeutic target for substance abuse. While some clinical studies have examined the ability of LTCC blockers to alter cocaine's subjective effects, very few LTCC studies have examined cocaine relapse. Here, we examined whether ventral tegmental area (VTA)-specific or systemic administration of the LTCC inhibitor, isradipine, altered cocaine-seeking behavior in a rat model. Male Sprague-Dawley rats first received 10 days of cocaine self-administration training ( $2 \mathrm{~h}$ sessions), where active lever depression resulted in delivery of a $\sim 0.5 \mathrm{mg} / \mathrm{kg}$ cocaine infusion paired with a tone + light cue. Rats then underwent 10 days of forced abstinence, without access to cocaine or cocaine cues. Rats were then returned to the opertant chamber for the cue-induced cocaine-seeking test, where active lever depression in the original training context resulted in tone + light cue presentation. We found VTA specific or systemic isradipine administration robustly attenuated cocaine-seeking, without altering cocaine-taking nor natural reward seeking. Dopamine (DA) signaling in the nucleus accumbens (NAC) core is necessary and sufficient for cue-induced drug-seeking. Surprisingly in our study, isradipine enhanced tonic and phasic DA signaling in cocaine abstinent rats, with no change in sucrose abstinent nor naïve rats. Strikingly, isradipine's behavioral effects were dependent upon NAc core DA receptor activation. Together, our findings reveal a novel mechanism by which the FDA-approved drug, isradipine, could act to decrease cocaine relapse.

Neuropsychopharmacology (2018) 43:2361-2372; https://doi.org/10.1038/s41386-018-0080-2

\section{INTRODUCTION}

Cue-induced drug craving and relapse is a component of substance abuse disorders that serves as a major barrier to successful cessation treatment [1-3]. Given the lack of FDA approved pharmacotherapies for cocaine addiction, there is great utility in identifying novel therapeutic targets. L-type calcium channels (LTCCS) and LTCC encoding genes are implicated in multiple neuropsychiatric disorders, including cocaine dependence [4-6]. Preclinical investigations in rodents have also revealed LTCC regulation of cocaine-related behaviors [7-9]. However, clinical and preclinical examinations of potential LTCC mechanisms that mediate drug craving and relapse are rather limited. Mechanistically, phasic dopamine (DA) signaling in the ventral tegmental area (VTA) to nucleus accumbens (NAc) pathway plays a critical role in drug-seeking and drug relapse behavior [10-12]. While LTCCs are known to regulate VTA neuronal firing patterns that mediate phasic DA signaling in the NAC [13-15], the role of LTCC regulation of DA signaling in drugrelapse remains unclear.

VTA LTCCs are known to play a critical role in mediating NAcdependent behavioral adaptations associated with cocaine exposure and abstinence $[5,8]$. However, previous preclinical VTA LTCC investigations were primarily performed with experimenter-delivered cocaine, where mechanisms may differ from those underlying cocaine self-administration and subsequent cocaine-seeking after forced or voluntary abstinence [16]. Here, we examined LTCC mechanisms in cue-induced cocaineseeking behavior after cocaine self-administration and forced abstinence in a preclinical rat model with strong face validity for human cue-induced craving and relapse [16]. We also performed in vivo voltammetry to determine whether LTCC blockade regulates DA signaling in the NAC of rats during forced cocaine abstinence. We used parallel, cue-induced sucrose-seeking experiments to determine if the LTCC blockade-induced behavioral and neurochemical effects were specific to cocaine, or also observed with a natural reward. Our results revealed the surprising finding that either VTA or systemic administration of the FDA approved LTCC blocker, isradipine, decreased cocaineseeking behavior while also increasing DA signaling in the NAc. In subsequent behavioral pharmacology experiments, we tested the causal relationship between isradipine's behavioral effects and the NAc dopaminergic effects. Together, our data suggest that the ability of isradipine to decrease cocaine-seeking is dependent upon DA receptor activity in the NAc. This new understanding of LTCC mechanisms that underlie cue-induced relapse has important implications for future clinical investigations and interventions.

\footnotetext{
${ }^{1}$ Department of Psychiatry, Yale School of Medicine, New Haven, CT 06511, USA; ${ }^{2}$ Department of Cellular and Molecular Physiology, Yale University, New Haven, CT 06511, USA; ${ }^{3}$ Interdepartmental Neuroscience Program, Yale University, New Haven, CT 06511, USA; ${ }^{4}$ Division of Pediatric Neurology, Department of Pediatrics, Weill Cornell Medical College of Cornell University, New York, NY 10065, USA and ${ }^{5}$ Feil Family Brain and Mind Research Institute, Weill Cornell Medical College of Cornell University, New York, NY 10065, USA Correspondence: Nii A. Addy (nii.addy@yale.edu)
}

Received: 12 January 2018 Revised: 18 April 2018 Accepted: 20 April 2018

Published online: 3 May 2018 


\section{MATERIALS AND METHODS}

Animals

Male Sprague Dawley rats (259-279 g; Charles River Laboratories, Wilmington, MA), housed 2 per cage prior to surgery and singly housed after surgery, were maintained under a $12 \mathrm{~h}$ light/dark cycle with climate control between $22-24^{\circ} \mathrm{C}$. Food and water were available ad libitum. All experiments were conducted according to the National Institutes of Health (NIH) Guide for the Care and Use of Laboratory Animals and were approved by the Yale University Institutional Animal Care and Use Committee (IACUC). In total, 138 rats were used for the behavioral experiments, with 13 rats excluded from the data analysis due to lack of acquired, stable self-administration behavior, non-patent i.v. catheters, or missedtargeted intracranial cannulae. Rats were assigned to experimental groups, in a counterbalanced fashion, based upon their selfadministration training. For in-vivo FSCV, 71 rats were used for data analysis, with 6 rats excluded due to a lack of stable evokedDA signals at baseline (4 rats), a missed placement (1 rat), or premature death during the voltammetry experiment (1 rat).

\section{Surgical procedures}

All surgical procedures were performed using aseptic techniques. Rats were anesthetized with ketamine $\mathrm{HCl}(100 \mathrm{mg} / \mathrm{kg}$, i.p., Sigma Aldrich, St. Louis, MO, USA) and xylazine $(10 \mathrm{mg} / \mathrm{kg}$, i.p., Sigma Aldrich, St. Louis, MO USA). Prior to surgical incision, rats received administration of the long-acting nonsteroidal anti-inflammatory drug, carpofen ( $5 \mathrm{mg} / \mathrm{kg}$ s.C., Henry Schein, Wallingford, CT, USA). Once rats reached an operational level of anesthesia, indwelling jugular catheters were implanted (Plastics One, Roanoke, VA, USA), as previously described [11]. Rats were then placed in a stereotaxic frame (David Kopf Instruments, Tujunga, CA, USA) for implantation of intra-cranial cannula. Coordinates were based on the rat brain atlas (Paxinos and Watson, 2007), with anteroposterior (AP), mediolateral (ML), and dorsoventral (DV) positions referenced from Bregma. For VTA infusion, a bilateral cannula spaced $1 \mathrm{~mm}$ apart (Plastics One, Roanoke, VA, USA) was placed $1 \mathrm{~mm}$ above the VTA (AP $-5.2 \mathrm{~mm}, \mathrm{ML} \pm 0.5 \mathrm{~mm}$, DV $-7.0 \mathrm{~mm}$ from dura). For NAc infusion, a bilateral cannula spaced $3 \mathrm{~mm}$ apart (Plastics One, Roanoke, VA, USA) was placed $1 \mathrm{~mm}$ above the NAc (AP +1.2 $\mathrm{mm}, \pm \mathrm{ML} 1.5 \mathrm{~mm}, \mathrm{DV}-5.5 \mathrm{~mm}$ from dura. All cannula were secured using screws (Gexpro, High Point, NC, USA) and dental cement (Dentsply, Milford, DE, USA). After surgery, rats were allowed to recover for 5-7 days before training.

\section{Cocaine and sucrose self-administration training}

Cocaine self-administration training was preceded by 2 to 3 days of food restriction to $\sim 90 \%$ of free feeding levels. Rats were trained under fixed ratio 1 (FR1) schedule of reinforcement, where active lever depression led to intravenous cocaine infusion $(0.18$ $\mathrm{mg}$ over $6 \mathrm{~s}, \sim 0.5 \mathrm{mg} / \mathrm{kg}$ ) and a conditioned stimulus (CS) presentation (tone + stimulus light for $6 \mathrm{~s}$, house light remained off), in a standard operant chamber (Med Associate, St. Albans, USA). Inactive lever depressions had no programmed consequences. For cocaine self-administration, each rat received $2 \mathrm{~h}$ sessions for 10 consecutive days. Acquisition of stable selfadministration behavior was defined as less than $15 \%$ variability in total active lever pressing over 3 consecutive days. Sucrose selfadministration training was similar, except that a $45 \mathrm{mg}$ sucrose pellet (Bio-Serve, Frenchtown, NJ) was delivered instead of cocaine, in a $1 \mathrm{~h}$ training session.

\section{Drug administration}

For intracranial drug infusion, bilateral internal cannula were inserted into the guide cannula and extended $1 \mathrm{~mm}$ beyond the guide cannula to target the VTA $(-8.0 \mathrm{~mm}$ from dura) or NAC $(-6.5 \mathrm{~mm}$ from dura). For behavioral and electrochemical experiments, drugs were delivered in a $0.3 \mu \mathrm{l}$ volume infused over $2 \mathrm{~min}$ via a micro-infusion pump and syringe (25 gauge,
Hamilton Syringe, Reno, NE, USA). After infusion, the internal cannulae were left in place for $1 \mathrm{~min}$ to allow for complete diffusion of the drug. The non-selective LTCC blocker, isradipine, (Sigma Aldrich, St. Louis, MO, USA) was dissolved in $1.7 \mathrm{mM}$ $\mathrm{EtOH}$. Doses of VTA ( $0 \mathrm{pg}$ to $223 \mathrm{pg}$ ) and systemic $(1.2 \mathrm{mg} / \mathrm{kg}$, i.p.) isradipine were based on previously published behavioral and physiologically effective doses [9]. The non-selective DA receptor antagonist, flupenthixol (Sigma Aldrich, St. Louis, MO, USA), was dissolved in $0.9 \%$ saline and infused at doses $(0 \mu \mathrm{g}$ to $20 \mu \mathrm{g})$ based on published data using a similar dose range for NAc core infusions [20].

Cue-induced cocaine or sucrose seeking test

After self-administration training, rats underwent 10 days of forced abstinence in their home cages, with no exposure to cocaine, or sucrose, and cues. On abstinence day 10, rats were placed in the original training operant chambers for $2 \mathrm{~h}$ for cocaine-seeking or $1 \mathrm{~h}$ for sucrose-seeking, where active lever depression led to the CS presentation alone (light + tone), with no cocaine or sucrose delivery. Inactive lever depressions had no programmed consequences. Intra-VTA or NAc infusions were given immediately prior to the cue-induced seeking session. For systemic drug experiments, i.p. injections were given 15 min before the cueseeking session, to allow time for drug bioavailability in the brain during the behavioral test.

In-vivo fast scan cyclic voltammetry

FSCV was used to measure dopamine fluctuations in the NAc. FSCV recordings were performed from abstinence days 10 to 13 in rats that had previously self-administered cocaine or sucrose. FSCV was also performed in cocaine and sucrose-naïve, age-matched controls. Rats were anesthetized with urethane $(1.5 \mathrm{~g} / \mathrm{kg}$, i.p.) and placed in a stereotaxic frame equipped with a heating pad to maintain body temperature (Harvard Apparatus, Holliston, MA, USA). A bipolar, stainless-steel stimulating electrode, coupled with a 26-gauge infusion cannula (Plastics One, Roanoke, VA, USA), was placed into the VTA (AP $-5.2 \mathrm{~mm}, \mathrm{ML}+0.5$ to $-1.5 \mathrm{~mm}$, DV between -7.4 and $-8.1 \mathrm{~mm}$ ), a carbon-fiber microelectrode was implanted in the NAc core (AP $+1.2 \mathrm{~mm}, \mathrm{ML}-1.4 \mathrm{~mm}$, DV from -6.0 to $7.0 \mathrm{~mm}$ ) and a $\mathrm{Ag} / \mathrm{Ag} \mathrm{Cl}$ reference electrode was placed in the contralateral hemisphere. A low-pass filtered $(2 \mathrm{kHz})$ triangular waveform $(-0.4$ to $+1.3 \mathrm{~V}$ and back to $-0.4 \mathrm{~V}$ at a rate of $400 \mathrm{~V} / \mathrm{s}$, repeated at $100 \mathrm{~ms}$ intervals) was applied to the carbon fiber microelectrode. Data were digitized and processed using NI-6711 and NI-6251 DAC/ADC cards (National Instruments, Austin, TX, USA) and Demon Voltammetry and Analysis Software (Wake Forest Baptist Medical Center, Winston-Salem, NC). Backgroundsubtracted cyclic voltammograms (CVs) were obtained by digitally subtracting stable background currents to resolve CVs for dopamine. Electrical pulses delivered to the VTA were computergenerated with a $6711 \mathrm{PCl}$ card (National Instruments, Austin, TX, USA) and were optically isolated from the electrochemical system (NL 800A, Neurolog, Digitimer Ltd, Hertfordshire, UK). Electrical stimulation for phasic evoked DA release consisted of biphasic square wave pulses $(300 \mu \mathrm{A}, 24$ pulses, with a $4 \mathrm{~ms}$ pulse width, applied at $60 \mathrm{~Hz}$ ). For tonic evoked DA release, all parameters remained the same except that the frequency was changed to 5 $\mathrm{Hz}$. VTA stimulation, and subsequent DA recordings in the NAC, were performed with a 3 min wait time between each stimulation to allow sufficient time for releasable DA stores to return to baseline, consistent with our previous work [17]. For the FSCV experiment, at baseline, 6 evoked-DA samples were first collected.

Histology

After the behavioral experiments, rats received a lethal dose of pentobarbital $(150 \mathrm{mg} / \mathrm{kg}$, i.p.) and $0.3 \mu \mathrm{L}$ of Chicago Blue Dye (Sigma Aldrich, St. Louis, MO, USA) was infused into the VTA or NAc. Coronal sections $(50 \mu \mathrm{m})$ were collected on a freezing stage 
microtome (Leica Microsystems, Bannockburn, IL) and mounted on glass microscope slides for observation. Rats with misplaced cannula or significant tissue damage were excluded from statistical analyses of the behavioral data. For voltammetry experiments, carbon fiber microelectrode placements were verified by applying a stepwise $10 \mu \mathrm{A}$ current to the carbon-fiber microelectrode, causing a small lesion at the recording site. Then, rats were given a lethal dose of pentobarbital $(150 \mathrm{mg} / \mathrm{kg}$, i.p.) and the brain was removed by dissection and stored in $3.2 \%$ formalin (Sigma Aldrich, St. Louis, MO, USA) for $48-72 \mathrm{~h}$. Next, brain tissue was sliced into $50 \mu \mathrm{m}$ sections on a stage-freezing microtome (Leica Microsystems, Bannockburn, IL), and lesions were assessed under a light microscope.

\section{Electrode calibration}

A subset of the carbon fiber microelectrodes, not used for lesions, were used to perform electrode calibration after the voltammetry experiment. Using a flow "t-cell" apparatus, electrodes were placed into the "t-cell" while known DA concentration (in Tris buffer) were presented at the electrode surface at a rate of $2 \mathrm{ml} /$ min. Calibration curves were created using DA concentrations of $250 \mathrm{nM}, 500 \mathrm{nM}$ and $1 \mu \mathrm{M}$ for each electrode. Current versus DA concentration curves were then averaged across all electrodes to obtain a line of best fit, and an equation that could be used to calculate DA concentration from obtained current.

\section{Statistics}

All behavioral data were analyzed using Graph Pad Prism 7 (Graph Pad Software, San Diego, CA) and SPSS 24 (IBM, Armonk, NY). Cocaine and sucrose self-administration training data were analyzed using a two-way repeated measures ANOVA, with lever (active vs. inactive) and day as factors. To determine if abstinence day 10 experimental groups differed in their prior selfadministration training, a two-way repeated measures ANOVA of "to-be" groups was performed with drug infusion (vehicle or drug) and training day as factors. For cue-induced seeking experiments and cocaine taking experiments, lever press data were analyzed using a two-way repeated measures ANOVA, with drug and lever as factors. For within-session time course analysis, data across the $60 \mathrm{~min}$ session was analyzed in $5 \mathrm{~min}$ bins. Then a two-way repeated measures ANOVA (drug and time as factors) was used to analyze lever press behavior. For cocaine taking experiments, analysis of total cocaine infusions was analyzed using an unpaired samples $t$-test. For analysis of the cue-induced cocaine-seeking experiment with combined systemic and NAC drug administration, a three-way repeated measures ANOVA was performed. Systemic drug (vehicle vs. isradipine), NAc drug (vehicle vs. flupenthixol) and lever (active vs. inactive) served as factors. For all behavioral analyses, if a two-way or three-way repeated measures ANOVA revealed significant main effects or a significant interaction $(p<$ $0.05)$, Bonferroni corrected post-hoc analyses were subsequently performed to compare between specific drug groups or doses.

Electrochemical data were analyzed using linear mixed models analyses in SPSS 24 (IBM, Armonk, NY). For each analysis, the covariate structure was explored and the model of best fit was chosen using the lowest Akaike information criterion value. The best fit covariate structure was determined to be $\operatorname{ARMA}(1,1)$ for the following experimental data: VTA drug effects on cue-induced cocaine-seeking, systemic drug (vehicle vs. isradipine) effects on phasic DA release in cocaine and sucrose abstinent rats (vehicle vs. isradipine), and phasic and tonic DA release in cocaine abstinent rats. For the systemic drug (vehicle vs. isradipine) effects on tonic evoked DA release in cocaine abstinent rats, the best fit covariate structure was factor analytic: first order. When significant interactions were found, Bonferroni post-hoc comparisons were used for additional comparisons between groups. For phasicevoked DA release in cocaine and sucrose abstinent rats, time was the repeated factor, and reward group (sucrose vs. cocaine) and
VTA drug (vehicle vs. isradipine) served as the additional factors. For tonic evoked DA release in cocaine abstinent rats, time was the repeated factor and VTA drug (vehicle vs. isradipine) was the additional factor. Phasic and tonic DA evoked release in cocaine abstinent rats was also measured when drug was given systemically, with time as a repeated factor and systemic drug (vehicle vs. isradipine) as the other factor.

\section{RESULTS}

VTA LTCC blockade selectively attenuates cue-seeking for cocaine We trained male Sprague-Dawley rats to self-administer cocaine ( $0.5 \mathrm{mg} / \mathrm{kg} /$ infusion, FR1 schedule) for ten days (Fig. 1a, Supplementary Fig. 1a), where rats readily learned to discriminate between the active and inactive levers (main effect of lever, $F_{1,28}$ $=71.64, p<0.001$, Supplementary Fig. 1a). Rats then underwent 10 days of forced abstinence and were tested for cue-induced cocaine seeking on day 20 (Fig. 1a). Rats receiving VTA vehicle or isradipine on day 20 (cue-induced cocaine-seeking test) did not show any a priori differences in active lever $\left(F_{2,26}=0.07, p>0.05\right.$, Fig. $1 \mathrm{~b})$ or inactive lever presses $\left(F_{2,26}=0.48, p>0.05\right.$, Fig. $\left.1 \mathrm{~b}\right)$ during training (days 1 through 10). On day 20, rats received bilateral, intra-VTA infusions of vehicle or isradipine $(0,74$ or $223 \mu \mathrm{g} / \mathrm{side}$, Fig. 1a,c) and were immediately tested for cueinduced cocaine-seeking, where each active lever press led to cue delivery (tone + stimulus light) in the absence of cocaine. Isradipine infusion led to a dose-dependent attenuation of cueseeking (main effect of drug, $F_{2,26}=6.00, p<0.01$; main effect of lever, $F_{1,26}=89.8, \quad p<0.0001 ;$ and a significant drug $\times$ lever interaction, $F_{2,26}=7.50, p<0.01$; post-hoc for active presses, 0 vs. $223 \mathrm{pg} / \mathrm{side}, p<0.0001$; post-hoc for active presses, 74 vs. $223 \mathrm{pg} / \mathrm{side}, p<0.01$, Fig. 1c). To examine cocaine reward, we tested whether VTA isradipine infusion would alter cocaine reinforcement in a separate cohort of rats. VTA LTCC blockade with isradipine immediately before the last cocaine selfadministration session (Day 10) did not alter the number of cocaine infusions received $\left(t_{13}=0.32, p>0.05\right.$, Fig. 1e) nor the number of active lever presses (no effect of VTA drug, $F_{1,13}=0.01$, $p>0.05$, Fig. 1e).

To determine if VTA LTCC effects on cue-seeking behavior were specific to cocaine or would also be observed with a natural reward, we trained a separate group of rats for sucrose selfadministration. Rats were trained to self-administer sucrose (45 mg pellet, FR1 schedule) for 10 days, followed by 10 days of forced abstinence (Fig. 2a, b, Supplementary Fig. 1b). Immediately prior to the sucrose-seeking test, isradipine was bilaterally infused into the VTA at a dose ( $223 \mathrm{pg} / \mathrm{side})$ that had effectively decreased cueinduced cocaine-seeking (Fig. 1c). Isradipine infusion failed to alter cue-induced sucrose-seeking (no effect of drug, $F_{1,12}=3.96$, $p>0.05$; main effect of lever, $F_{1,12}=48.46 ; p<0.0001$; and no drug $x$ lever interaction, $F_{1,12}=0 ; p>0.05$, Fig. $2 c$ ). We note that sucrose self-administration training produced high rates of responding (200 to 400 lever presses per session). Indeed, on day 6 of training, rats showed decreased active lever responding, compared to day 5 (main effect of day, $F_{9,162}=9.10, p<0.0001$; post hoc active presses for day 5 vs. day $6, p<0.01$, Fig. 2b), perhaps due to the large amounts of total sucrose procured during days 1 through 5 of training. To control for potential confounds of this one day decrease in responding, we performed an additional self-administration experiment where active levers were retracted for a $6 \mathrm{~s}$ timeout during each sucrose pellet delivery (Fig. 2d). Here, self-administration acquisition curves (Fig. 2d, Supplementary Fig. 1c) were similar to those observed for cocaine (Fig. 1b), although with a higher magnitude of responding for sucrose training. Again, VTA isradipine did not alter cueinduced sucrose-seeking behavior (no effect of drug, $F_{1,12}=$ 0.6103, $p>0.05$, main effect of lever, $F_{1,12}=75.44 ; p<0.0001$ and no drug $\times$ lever interaction, $F_{1,12}=0 ; p>0.05$, Fig. 2e). Together, 
a.
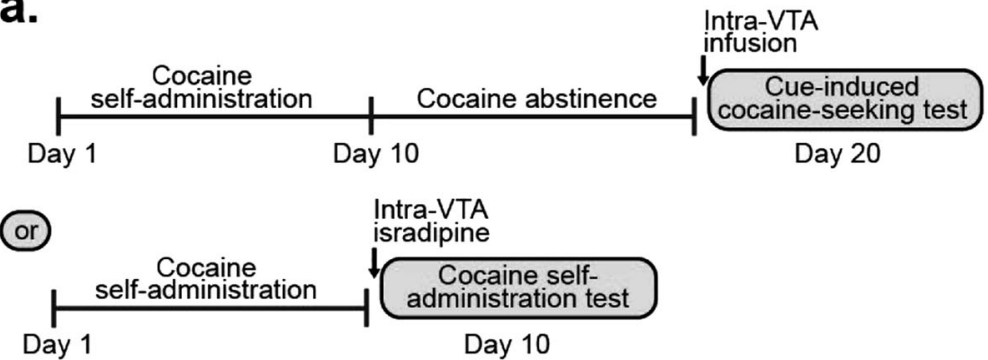

b.

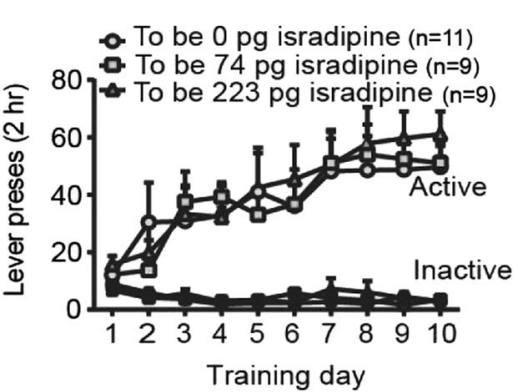

d. Cocaine self-administration

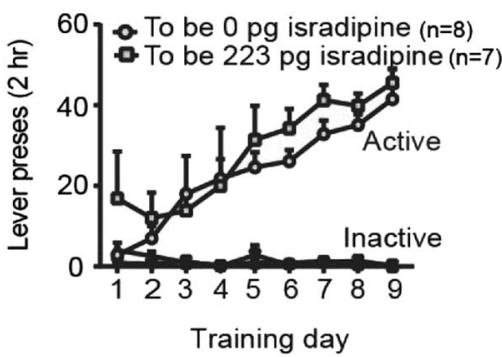

C. Cue-induced cocaine-seeking test

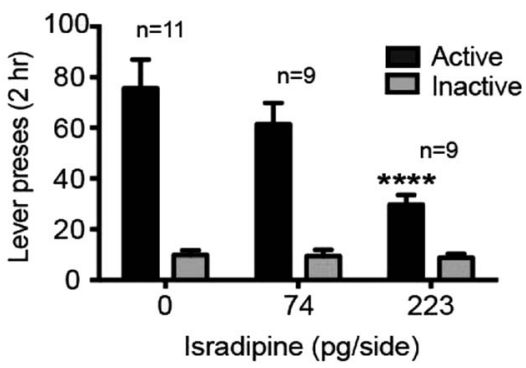

e. Day 10 cocaine taking behavior

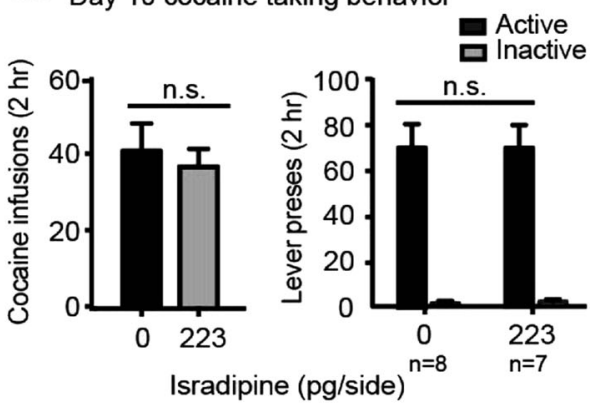

f. Histology

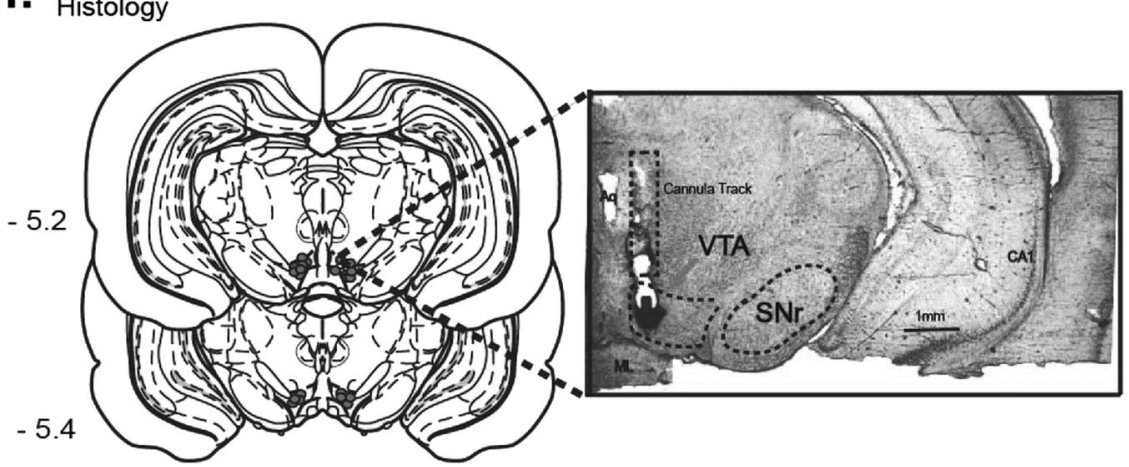

Fig. 1 Intra-VTA isradipine attenuates cue-induced cocaine-seeking, but not cocaine-taking. a Experimental timeline for cue-induced cocaineseeking and cocaine taking experiments. b Day 1 to 10 cocaine self-administration training, where rats did not show a-priori differences in active or inactive lever presses based on their experimental ("to be") assignment for the cocaine-seeking test. c Intra-VTA isradipine administration, on abstinence day 10, dose-dependently decreased cue-induced cocaine-seeking behavior. d Day 1 to 9 cocaine selfadministration training, where rats showed no a-priori differences in active or inactive lever presses, based on day 10 ("to-be") experimental assignment. e Intra-VTA isradipine administration on cocaine-taking day 10 did not alter the number of cocaine infusions and did not alter the number of lever presses. $f$ Histological verification of VTA cannula placements for cocaine experiments. Data are presented as \pm the standard error of the mean (SEM). ${ }^{* * *} p<0.0001$ (active lever presses for 0 vs. $223 \mathrm{pg} /$ side isradipine, post-hoc analysis with a Bonferroni correction)

these data demonstrate that acute VTA LTCC blockade after 10 days of abstinence selectively attenuates reward-seeking mediated by cocaine-paired cues, and not natural reward sucrose-paired cues.
VTA LTCC blockade selectively enhances phasic DA signaling in cocaine abstinent rats

Phasic dopamine (DA) signaling in the nucleus accumbens core (NAc) is necessary and sufficient for cue-induced cocaine seeking 
a. Intra-VTA

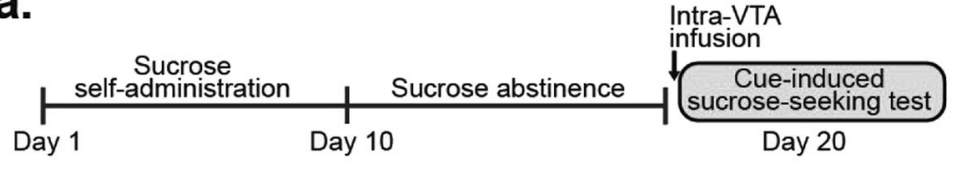

b.

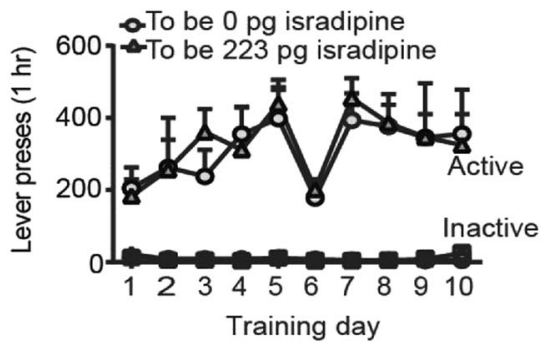

d. Sucrose self-administration

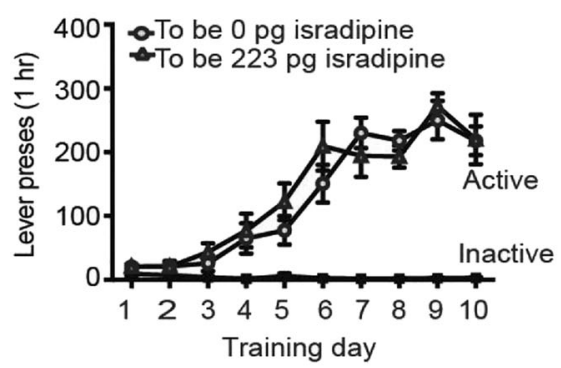

C. Cue-induced sucrose-seeking test

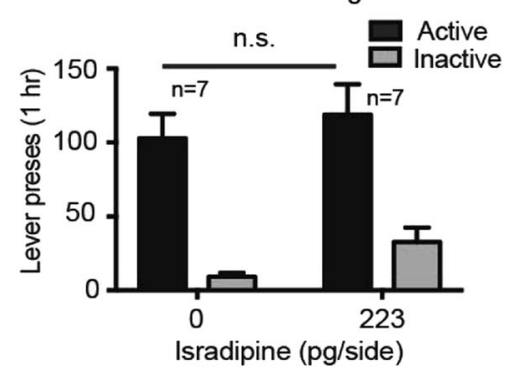

e. Cue-induced sucrose-seeking test

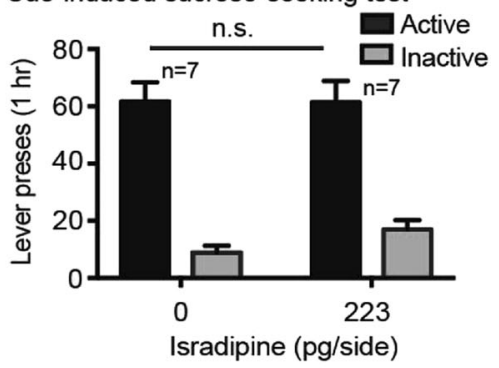

f.

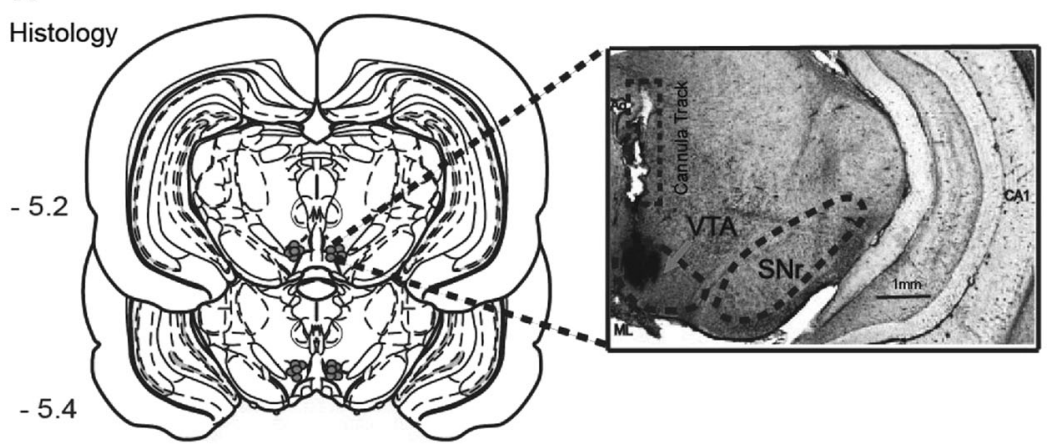

Fig. 2 Intra-VTA isradipine does not alter cue-induced sucrose-seeking behavior. a Experimental timeline for cue-induced sucrose-seeking experiments. b Day 1 to 10 sucrose self-administration training, represented based on subjects ("to be") experimental assignment for the subsequent sucrose-seeking test. Active levers were continuously available in the operant chamber throughout the training sessions. c VTA infusion of isradipine on abstinence day 10 did not alter cue-induced sucrose-seeking behavior. d Day 1 to 10 sucrose self-administration training based on subjects subsequent abstinence day 10 ("to-be") experimental assignment. During self-administration training, each sucrose pellet delivery was followed by active lever retraction for a $6 \mathrm{~s}$ timeout period. e VTA isradipine infusion on abstinence day 10 again did not alter cue-induced sucrose-seeking behavior. $\mathbf{f}$ Histological verification of VTA cannula placements for sucrose experiments. Data are presented as \pm the SEM

[10-12]. Moreover, we previously found that VTA cholinergic receptor blockade, which attenuates cue-induced cocaine seeking, also attenuates phasic DA release in the NAc core [11]. Here, we sought to determine whether VTA LTCC blockade also decreased phasic DA signaling in cocaine abstinent subjects. Rats were trained to self-administer cocaine or sucrose for 10 days, followed by 10 days of forced cocaine or sucrose abstinence (Fig. 3a). Rats were then anesthetized with urethane $(1.5 \mathrm{mg} / \mathrm{kg}$, intraperitoneal, i.p.) and prepared for in vivo fast scan cyclic voltammetry (FSCV), as previously described $[11,17]$. FSCV was performed over a range of 10 to 13 days of abstinence, due to practical limitations regarding how many voltammetry experiments could be performed in a single day. Evoked phasic dopamine signaling in the NAC core was examined following phasic-like $(300 \mu \mathrm{A}$, 24 pulse, $60 \mathrm{~Hz}$ ) stimulation of the VTA (Fig. 3b, c). Analysis revealed that VTA LTCC blockade differentially influenced NAC phasic DA release, over one hour of recording, in cocaine abstinent versus sucrose abstinent rats (main effect of reward group $F_{1,21}=9.174, p<0.01$; main effect of VTA drug, $F_{1,21}=7.173$, $p<0.05$; main effect of time, $F_{20,183}=2.68 ; p<0.001$; and significant reward group $\times$ VTA drug $\times$ time interaction, $F_{20,183}=$ 1.934; $p=0.01$, Fig. $3 d$ ). Surprisingly in cocaine abstinent rats, VTA LTCC blockade led to enhanced evoked phasic DA concentrations, compared to VTA vehicle infusion (significant effect of VTA drug, 
a.

\begin{tabular}{|c|c|c|}
$\begin{array}{c}\text { Cocaine or sucrose } \\
\text { self-administration }\end{array}$ & $\begin{array}{c}\text { Cocaine or sucrose } \\
\text { abstinence }\end{array}$ & FSCV \\
Day 1 & Day 10 & Day $20-23$
\end{tabular}

b.

NAc phasic DA signaling - cocaine abstinence
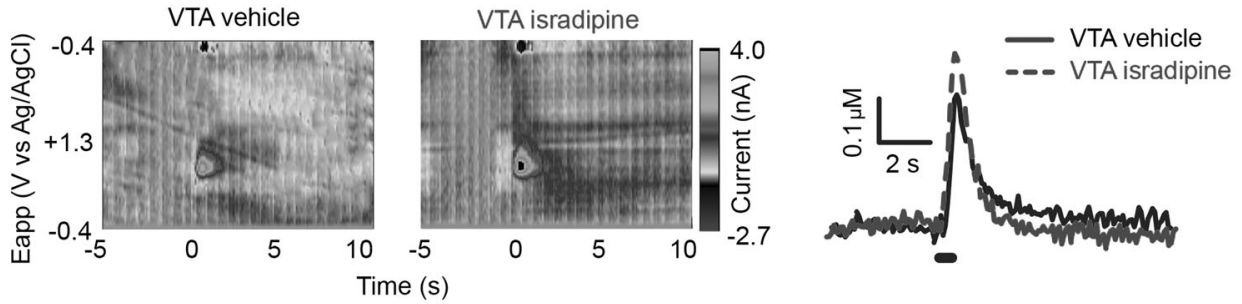

C.

NAc phasic DA signaling - sucrose abstinence

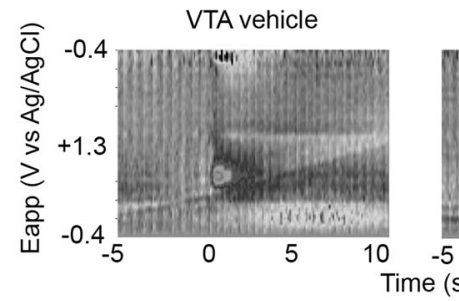

VTA isradipine
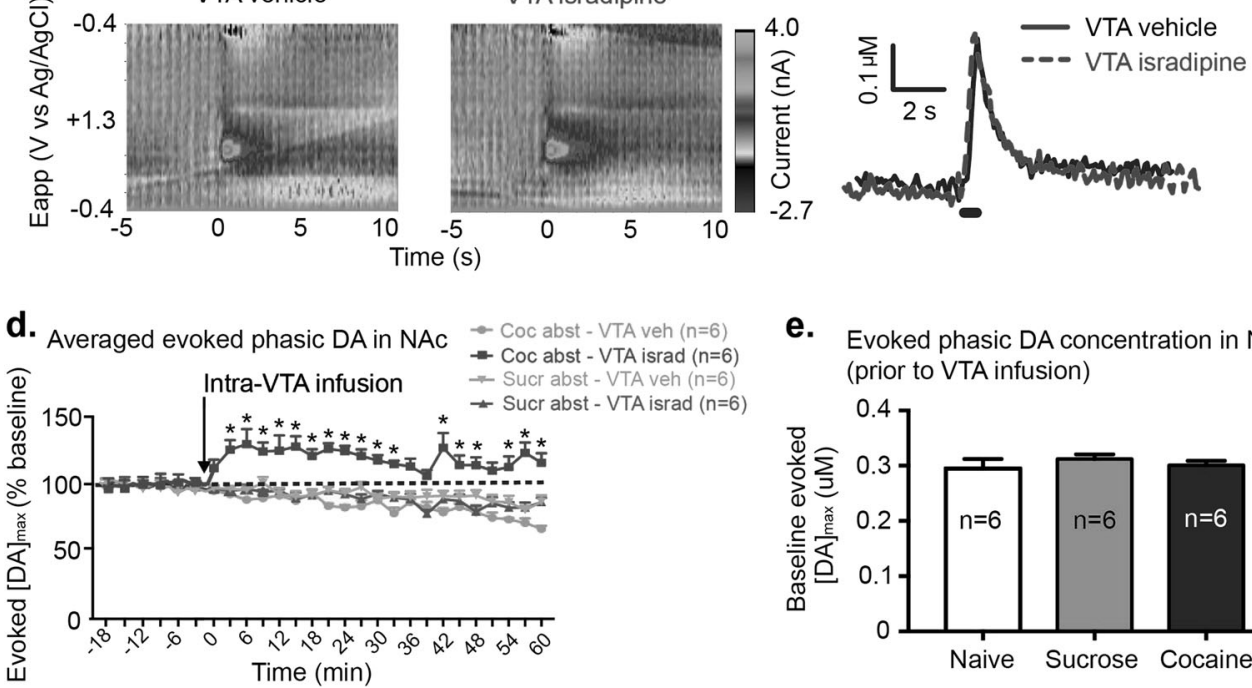

e. Evoked phasic DA concentration in NAc (prior to VTA infusion)

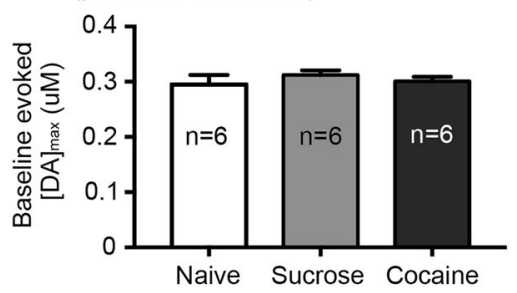

Fig. 3 Intra-VTA isradipine enhances phasic dopamine (DA) signaling in the NAc of cocaine abstinent, but not sucrose abstinent, rats. a Experimental timeline for cocaine or sucrose self-administration experiments and subsequent DA analysis with FSCV. b Representative current vs. time color plots (left) and DA concentration vs. time traces (right) in cocaine abstinent rats. c Representative current vs. time color plots (left) and DA concentration vs. time traces (right) in sucrose abstinent rats. d Peak evoked phasic DA release in cocaine abstinent and sucrose abstinent rats. Data are represented as a percentage, compared to the average peak evoked DA concentrations in 6 pre-infusion samples. Intra-VTA isradipine led to increased peak phasic DA release in cocaine-abstinent rats, compared to other groups. e Peak phasic evoked DA release in the NAc of naïve, sucrose abstinent and cocaine abstinent rats prior to VTA infusion. Analysis revealed no differences in phasic DA release. Data are presented as \pm the SEM. ${ }^{*} p<0.05$ (significant difference between VTA vehicle and VTA isradipine infusion in cocaine abstinent rats, Bonferroni corrected comparisons)

within the cocaine group at time points 3-33, 42-48, 54-60, $p<$ 0.05; Fig. 3d). In sucrose abstinent rats, in contrast, VTA LTCC blockade did not alter evoked phasic DA compared to VTA vehicle (no effect of VTA drug, within the sucrose group at time points $0-60, p>0.05$; Fig. 3d). Furthermore, VTA LTCC blockade did not alter phasic DA release in a separate cohort of age-matched control rats, that were naïve to both cocaine and sucrose (no effect of VTA drug, $F_{1,20}=0.171 ; p>0.05$, Supplementary Fig. 3). As an additional control, we compared baseline evoked phasic DA concentrations in naïve, sucrose abstinent, and cocaine abstinent rats prior to VTA isradipine infusion. The data revealed no differences in peak evoked phasic DA release concentrations between naïve, sucrose abstinent, and cocaine abstinent rats (no main effect of group, $F_{2,33}=0.413, p>0.05$, Fig. 3e). Thus, the increase in evoked phasic DA signaling was only observed in cocaine abstinent rats, after VTA LTCC blockade.

Given the unexpected increase in evoked phasic DA release in cocaine abstinent rats, we examined whether such effects were specific to phasic DA or reflective of an increase in overall DA tone. We used tonic-like stimulation of the VTA $(300 \mu \mathrm{A}, 24$ pulse, $5 \mathrm{~Hz})$ to determine the effect of VTA LTCC blockade on tonic-evoked DA signaling in the NAc core of cocaine abstinent rats. Our stimulation frequency $(5 \mathrm{~Hz})$ was consistent with DA tonic firing observed in vivo $(3-7 \mathrm{~Hz})$ [18] and similar to tonic-like stimulation parameters used in previous in vivo voltammetry work [19]. In cocaine abstinent rats, VTA LTCC blockade led to greater tonicevoked DA release compared to VTA vehicle (Fig. 4a, main effect of VTA infusion, $F_{1,10}=31.74, p<0.001$, Fig. 4b). The observed difference between vehicle and isradipine may have been partially due to a DA decrease in the vehicle group (Fig. 4b). Indeed, it is possible that the presence of $1.7 \mathrm{mM} \mathrm{EtOH}$ in the vehicle could decrease DA levels. Thus, to more specifically examine VTA isradipine effects, we performed a within-subject comparison of averaged peak tonic evoked DA between the 6 pre-VTA infusion DA recordings and the 6 post-VTA infusion DA recordings (Fig. 4c). Importantly, the 6 post-infusion DA recordings (collected over 18 
a.

NAc tonic DA signaling - cocaine abstinence
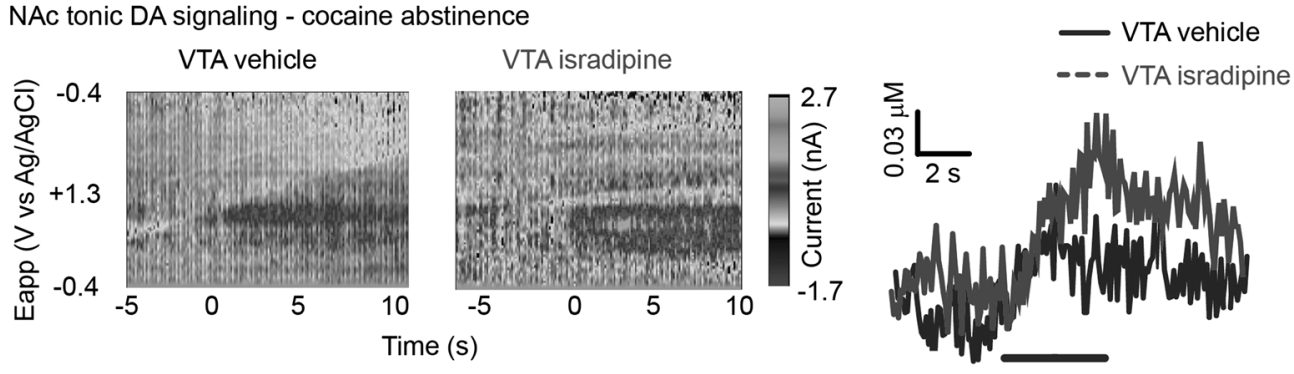

b. Averaged evoked tonic DA in NAc

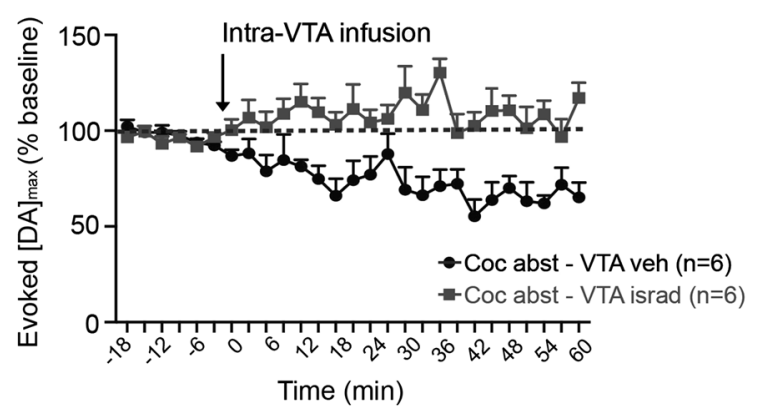

C. Tonic DA during cocaine abstinence

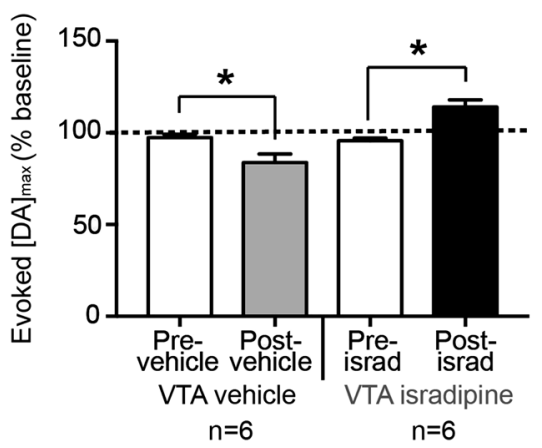

Fig. 4 Intra-VTA isradipine increases tonic DA signaling in the NAc. a Representative current vs. time color plots (left) and DA concentration vs. time traces (right) in cocaine abstinent rats. b Peak evoked tonic DA concentrations in cocaine abstinent rats. Data are represented as a percentage, compared to the average peak evoked DA in 6 pre-infusion samples. During cocaine abstinence, VTA isradipine-infused rats showed higher peak evoked tonic DA release compared to VTA vehicle infused rats. c Within-subject comparisons of 6 pre-VTA infusion tonic DA release to 6 post-VTA infusion tonic DA release. During cocaine abstinence, VTA vehicle infusion led to significant decrease in evoked tonic DA, while VTA isradipine infusion led to a significant increase in evoked tonic DA. Data are presented as \pm the SEM. ${ }^{*} p<0.05$ (pre vs. post vehicle infusion or pre vs. post-isradipine infusion, paired samples $t$-test)

min) corresponds to the post-infusion time frame (first $20 \mathrm{~min}$ ) where VTA isradipine infused rats showed the most pronounced attenuation of cue-induced cocaine-seeking (main effect of VTA drug on active lever presses, $F_{1,18}=15.9, p<0.001$, significant post-hoc differences between VTA vehicle and isradipine at time bins $5,10,15$, and 20, $p<0.0001, p<0.0001, p<0.01$, and $p<0.05$ respectively, Supplementary Fig. 2a). Indeed, VTA vehicle infused rats showed a significant decrease in tonic DA, compared to preinfusion DA levels $\left(t_{5}=3.374, p<0.05\right.$, Fig. $\left.4 c\right)$. In contrast, VTA isradipine rats showed a significant increase in tonic DA after isradipine infusion $\left(t_{5}=4.95 ; p<0.01\right.$, Fig. $\left.4 c\right)$. Thus, despite the decrease observed after vehicle infusion in cocaine abstinent rats, VTA isradipine infusion in separate cocaine abstinent rats still produced a significant increase in tonic DA over pre-infusion baseline levels.

Systemic isradipine administration also decreases cue-induced cocaine seeking and elevates NAc DA

Given the ability of isradipine to decrease cocaine-seeking after VTA administration, we sought to determine whether systemic isradipine administration would also decrease cue-induced cocaine-seeking. Such investigations have important clinical implications, given the current clinical use of systemically administered, oral isradipine [6]. Rats underwent 10 days of cocaine self-administration (Fig. 5a, b), followed by 10 days of forced abstinence and a cue-induced cocaine-seeking test (Fig. 5a). Systemic vehicle or isradipine was administered $15 \mathrm{~min}$ prior to the cocaine-seeking test, to allow time for systemically administered drug to reach the brain. Systemic isradipine $(1.2 \mathrm{mg} / \mathrm{kg}$, i.p.) significantly decreased cue-induced cocaine-seeking (main effect of drug $F_{1,13}=11.42, p<0.05$; significant drug $\times$ lever interaction $F_{1,13}=17.79 ; p<0.05$, Fig. $5 c$; post-hoc significant decrease in active lever between vehicle and isradipine, $p<0.05$, Fig. $5 c$ ). We subsequently determined if systemic isradipine also altered NAC DA signaling during cocaine abstinence. Compared to vehicle, systemic administration of isradipine $(1.2 \mathrm{mg} / \mathrm{kg}$, i.p.) increased evoked phasic DA release (main effect of drug, $F_{1,11}=28.56 ; p<$ 0.001 , no effect of time, $F_{1,20}=1.618, p>0.05$, and strong trend for drug $\times$ time interaction, $F_{20,100}=1.66 ; p=0.054$ Fig. $5 d$ ) -an effect that emerged 12 min after systemic injection (significant DA difference between isradipine and vehicle groups at time points 12-15, 21-56, all $p<0.05$; Fig. 5d). Systemic isradipine also increased evoked tonic DA release (main effect of drug, $F_{1,9}=$ $15.96, p<0.01$; main effect of time, $F_{20,71}=1.89, p<0.05$; significant drug $\times$ time interaction, $F_{20,71}=2.63, p<0.01$; Fig. 5e) in the NAc. The systemic isradipine effect on tonic evoked DA also emerged starting $12 \mathrm{~min}$ after injection (significant DA difference between isradipine and vehicle groups at $12,15,24,36-42,48,51$, and $60 \mathrm{~min}$, all $p<0.05$, Fig. 5e). This emergence of phasic and tonic DA effects 12 to $15 \mathrm{~min}$ after systemic isradipine corresponds to our analysis time frame for cocaine-seeking behavior $15 \mathrm{~min}$ after systemic isradipine where we observed a robust attenuation of cocaine-seeking behavior (main effect of systemic drug on active lever presses, $F_{1,13}=16.4, p<0.001$, significant post-hoc differences between systemic vehicle and systemic isradipine at time bins 5, 10, 15, 20, and 35, $p<0.0001, p<0.0001, p<0.0001, p$ $<0.01$ and $p<0.05$ respectively, Supplementary Fig. 2 b).

DA receptor activity in the NAc core is required for systemic isradipine-induced decreases in cocaine-seeking

Given the ability of LTCC blockade to decrease cocaine-seeking and increase DA signaling, we sought to determine whether these behavioral effects were mediated by increased DA activity in the NAc. With our observed isradipine-induced increases in NAc DA, 
a.

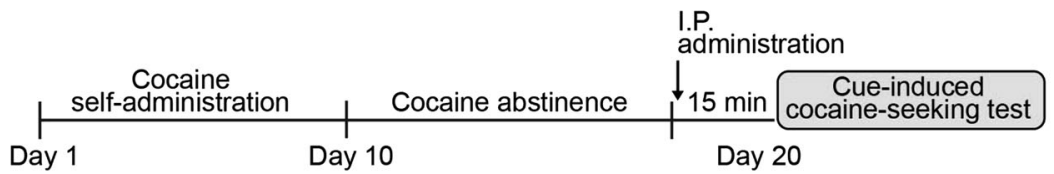

or

\begin{tabular}{|c|c|c|}
$\begin{array}{c}\text { Cocaine } \\
\text { self-administration }\end{array}$ & Cocaine abstinence & FSCV \\
Day 1 & Day 10 & Day $20-23$
\end{tabular}

b.

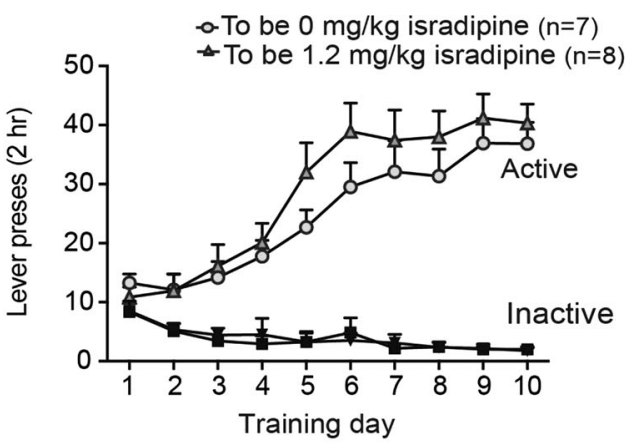

d. Averaged evoked phasic DA in NAC

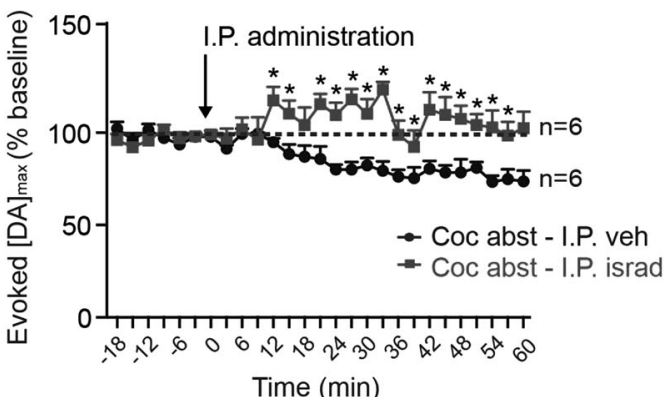

C. Cue-induced cocaine-seeking test

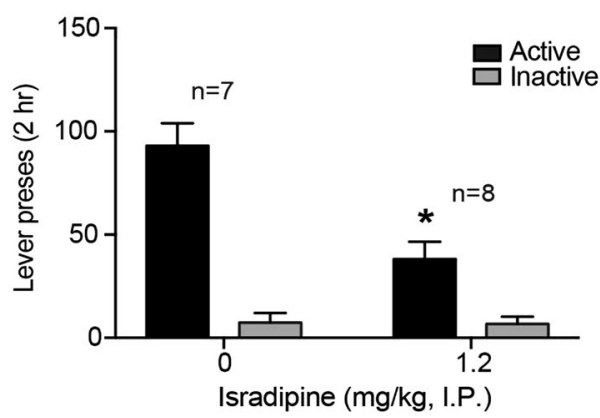

e. Averaged evoked tonic DA in NAC

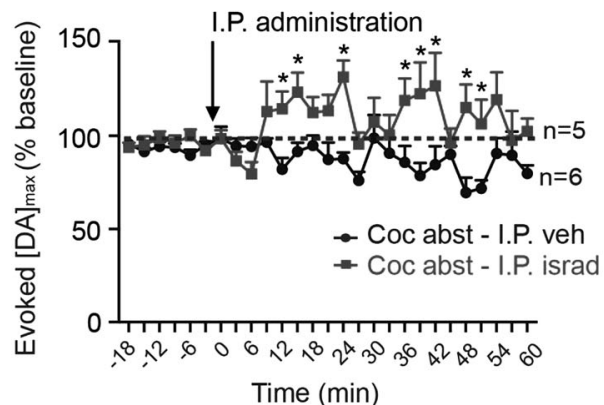

Fig. 5 Systemic isradipine administration attenuates cue-induced cocaine-seeking and enhances NAc DA signaling. a Timeline of systemic isradipine experiments for cue-induced cocaine-seeking and voltammetric analysis of DA signaling in the NAc. b Day 1 to 10 cocaine selfadministration training, based on "to be" experimental assignments for the cue-induced cocaine-seeking test. c Systemic administration of isradipine, on abstinence day 10, significantly attenuated cue-induced cocaine-seeking. $\mathbf{d}$ Average evoked phasic DA release in the NAc of cocaine abstinent rats. Systemic isradipine administration led to significantly greater phasic DA release, compared to systemic vehicle administration. e Averaged evoked tonic DA release in the NAc of cocaine abstinent rats. Systemic isradipine also led to significantly greater tonic DA release, compared to systemic vehicle. Data are presented as \pm the SEM. ${ }^{*} p<0.05$ (panel c: post-hoc significant decrease in active lever presses for vehicle vs. isradipine rats, Bonferroni corrected comparisons, panels $\mathbf{d}$ and e: post-hoc significant DA differences in vehicle vs. isradipine rats, Bonferroni corrected comparisons)

we hypothesized that activation of NAc core DA receptors would be required for the isradipine-induced decrease in cocaineseeking. Our experimental goal was to pharmacologically inhibit NAc core DA receptors to determine if this would disrupt isradipine's behavioral effects. However, previous work had shown that NAc core infusion of a DA receptor antagonist, flupenthixol, robustly attenuated cue-induced cocaine-seeking [20]. Thus, we sought to determine whether NAc infusion of a subthreshold flupenthixol dose, with minimal effects on its own, could block systemic isradipine's behavioral effects. In our flupenthixol dose response experiment, flupenthixol infusion into the NAc core led to a dose-dependent decrease in cue-induced cocaine-seeking (main effect of drug, $F_{2,21}=31.33, p<0.001$, and a significant drug $\times$ lever interaction $F_{2,21}=19.0 ; p<0.001$, Fig. 6c). However, the $7 \mu \mathrm{g} / \mathrm{side}$ flupenthixol dose did not alter active nor inactive lever presses compared to the vehicle group (all post-hoc comparisons, $p>0.05$, Fig. $6 c$ ). Thus, we next examined whether
NAc core infusion of the subthreshold $7 \mu \mathrm{g} /$ side flupenthixol dose would alter the ability of systemic isradipine to decrease cueinduced cocaine seeking. The results revealed differences in the systemic isradipine effects, in the presence versus absence of NAc flupenthixol (main effect of systemic isradipine $F_{1,72}=38.905, p<$ 0.001 ; no main effect of NAc flupenthixol $F_{1,72}=.107, p>0.05$; and a significant systemic drug $\times$ NAc drug $\times$ lever interaction, $F_{1,72}=53.683, p<0.001$ Fig. 6 f). Thus, systemic administration of isradipine significantly decreased cue-induced cocaine-seeking (main effect of systemic isradipine $F_{1,72}=38.905, p<0.001$, posthoc comparison of active lever presses in systemic vehicle plus NAc vehicle versus systemic isradipine plus NAc vehicle, $p<0.01$, Fig. 6f). In contrast, rats receiving systemic isradipine plus NAC flupenthixol showed significantly more active lever presses compared to rats receiving isradipine alone (post hoc comparisons, $p<0.01$, Fig. 6f). In addition, rats receiving systemic isradipine plus NAc flupenthixol did not differ from rats receiving 
a.

\begin{tabular}{|c|c|c|}
\hline & & $\begin{array}{l}\text { Intra-NAc } \\
\text { infusion }\end{array}$ \\
\hline $\begin{array}{l}\text { Cocaine } \\
\text { self-administration }\end{array}$ & Cocaine abstinence & $\begin{array}{c}\text { Cue-induced } \\
\text { cocaine-seeking test }\end{array}$ \\
\hline Day 1 & ay 10 & Day 20 \\
\hline
\end{tabular}

b.

Cocaine self-administration

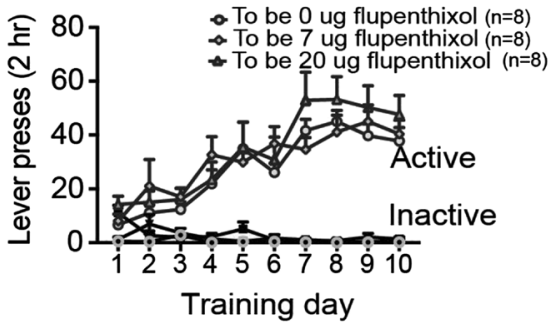

C. Cue-induced cocaine-seeking test

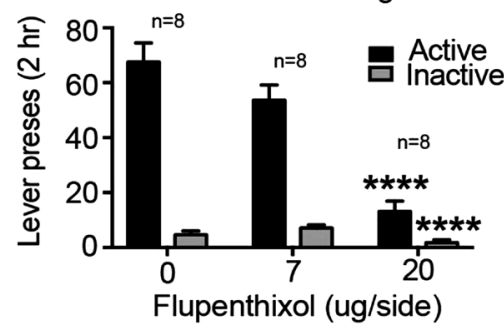

d.

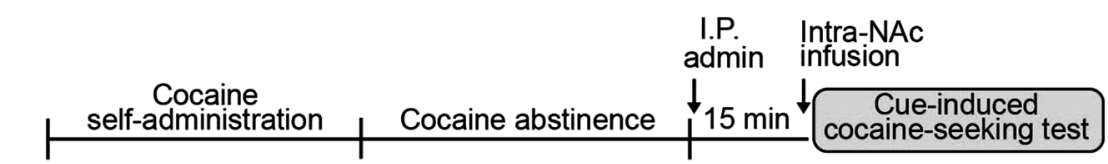

Day 1

Day 10

Day 20

e. Cocaine self-administration

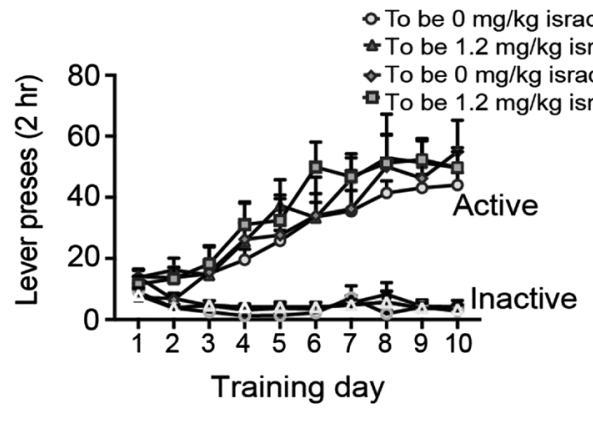

f.

f. Cue-induced cocaine-seeking test

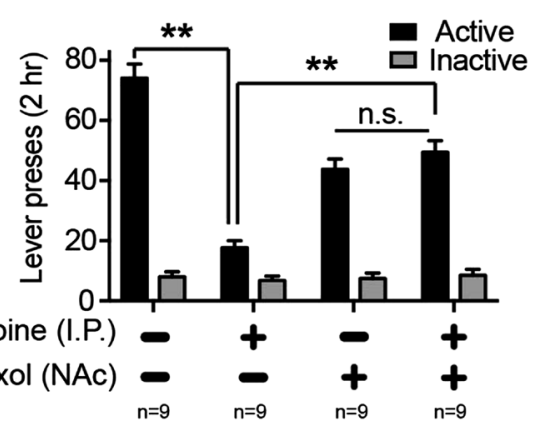

g.

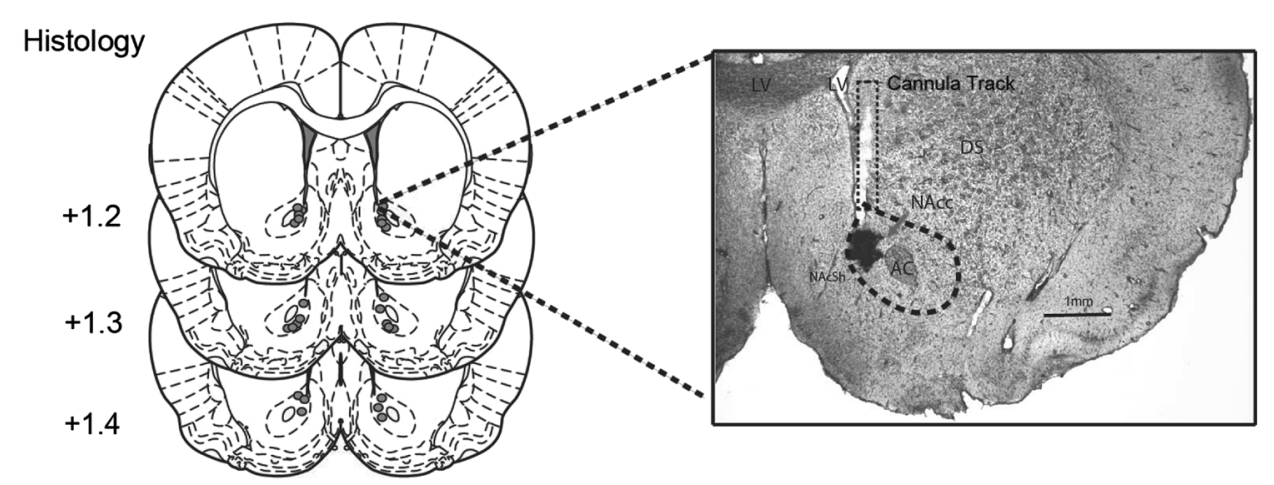

Fig. 6 DA receptor antagonism in the NAc blocks the behavioral effects of systemic isradipine. a Timeline for intra-NAc flupenthixol experiments with cue-induced cocaine-seeking. b Day 1 to 10 cocaine self-administration training based on "to be" experimental assignment for the cue-induced cocaine-seeking test. c Intra-NAc infusion of the DA receptor antagonist, flupenthixol, dose-dependently decreased lever press activity during the cue-induced cocaine-seeking test. $\mathbf{d}$ Experimental timeline for combined systemic isradipine and NAc flupenthixol administration prior to cue-induced cocaine-seeking test. e Day 1 to 10 cocaine self-administration training based on abstinence day 10 , "to be" experimental assignment. f Systemic administration of isradipine on abstinence day 10 significantly attenuated cue-induced cocaineseeking. Systemic isradipine differentially influenced cocaine-seeking behavior in the presence versus absence of NAc flupenthixol. In presence of NAc flupenthixol, systemic isradipine did not alter cocaine-seeking behavior. In contrast, rats receiving systemic isradipine alone showed decreased cocaine-seeking compared to rats with systemic isradipine plus NAc flupenthixol. Data are presented as \pm the SEM. ${ }^{* *} p<$ $0.01,{ }^{* * * *} p<0.001$ (panel c, Bonferroni corrected post-hoc comparisons of active lever presses for $20 \mu \mathrm{g} / \mathrm{side}$ flupenthixol vs. 0 or $7 \mu \mathrm{g} / \mathrm{side}$. Bonferroni corrected post-hoc comparison of inactive lever presses for $20 \mu \mathrm{g} / \mathrm{side}$ flupenthixol vs. 0 or $7 \mu \mathrm{g} / \mathrm{side}$. $\mathrm{g}$ Histological verification of NAc cannula placements 
systemic vehicle and NAc flupenthixol (post hoc comparisons, $p>$ 0.05 Fig. 6f). Thus, isradipine administration in the presence of $7 \mu \mathrm{g}$ flupenthixol no longer decreased cocaine-seeking, reflected in cocaine-seeking behavior that was same as the flupenthixol alone group. Importantly, this lack of an isradipine-induced decrease, when combined with $7 \mu \mathrm{g}$ flupenthixol, was not due to a floor effect, given the ability of $20 \mu \mathrm{g}$ flupenthixol to further decrease active lever responding (Fig. $6 \mathrm{c}$ ). While there is a possibility for serotonin contributions, due to flupenthixol actions at 5HT2 receptors [21], collectively our behavioral and electrochemical data strongly suggest that the isradipine-induced decrease in cocaine seeking is dependent upon NAc core DA receptor activity.

\section{DISCUSSION}

Very few studies have investigated LTCC inhibitor effects on cocaine relapse, nor the mechanistic underpinnings by which LTCC blockade may alter such relapse. Our study reveals a novel mechanism through which acute administration of isradipine, an FDA approved medication, attenuates cue-induced cocaineseeking. We find that VTA isradipine infusion selectively attenuates cue-induced cocaine-seeking, without altering cocaine reinforcement nor cue-induced sucrose-seeking. Surprisingly, isradipine administration in cocaine abstinent rats also increases tonic and phasic-evoked DA in the NAc. The behavioral and dopaminergic effects of VTA isradipine infusion are also observed following systemic isradipine administration. Indeed, the isradipine-induced increase in NAc DA appears to mediate isradipine's behavioral effects, as DA receptor antagonism in the NAc blocks the ability of isradipine to decrease cocaine-seeking.

VTA to NAC LTCC mechanisms mediating drug-related behavior Our findings add to the emerging evidence of VTA to NAC LTCC mechanisms in behaviors associated with repeated cocaine exposure and abstinence $[8,9]$. The unexpected increase in dopamine during cocaine abstinence is opposite of what one would predict based on prior electrophysiological, electrochemical and behavioral data. Indeed, LTCC activation in the VTA is known to induce DA burst firing [13], and the Cav1.3 LTCC subtype is known to facilitate the transition from tonic to burst DA firing [14]. Furthermore, LTCC inhibition with isradipine blocks LTP induction and reverses previously established LTP in VTA DA neurons [9]. However, the effects of LTCC manipulation on DA activity and signaling during cocaine abstinence were unknown prior to our study. Based on the ability of LTCC inhibition to decrease DA burst activity, we hypothesized that LTCC inhibition would attenuate phasic DA signaling. Although the isradipine-induced increase in dopamine is opposite of our original hypothesis, the effect is consistent with previous ethanol findings, where systemic administration of an LTCC inhibitor, nimodipine, increased extracellular dopamine levels, as measured by microdialysis [22]. Nimodipine reversed both the ethanol-withdrawal induced DA deficit and reduced ethanol-induced behavioral withdrawal [22]. These LTCC dopaminergic effects were specific to ethanol, and were not observed in sucrose withdrawn rats-consistent with our drug-specific isradipine-induced DA enhancement in cocaineexposed, but not sucrose-exposed, subjects [22]. An important implication of these findings is the future identification of the mechanism by which LTCC blockade increases DA.

While VTA LTCCs are located post-synaptically on DA cell bodies [5, 23], it is possible that isradipine's dopaminergic effects are instead mediated by pre-synaptic LTCCS. Recent evidence revealed the ability of LTCC activation and blockade in the substantia nigra reticulata to increase and decrease GABA release, respectively-effects suggested to be mediated by LTCCs on GABA terminals [24]. If presynaptic LTCCs are also present on GABA terminals in the VTA, one would predict that isradipine infusion could decrease VTA inhibitory tone to facilitate enhanced
DA activity and downstream DA release. Additional mechanistic insight could be gained by comparing VTA LTCC expression in cocaine versus sucrose abstinent rats. It would also be informative to directly examine DA responses to cues, after isradipine administration. However, these mechanistic questions have yet to be examined. Another key consideration is the mechanisms by which increased dopamine leads to decreased cocaine-seeking. Decreases in DA levels have been previously observed during cocaine abstinence [25], providing evidence for the DA depletion hypothesis of cocaine abstinence $[26,27]$. It is possible that isradipine-induced increases in dopamine act to "reverse" the cocaine abstinence-induced DA depletion. We did not include microdialysis examination of basal DA levels during cocaine abstinence here. However, future microdialysis experiments could be used to determine whether cocaine abstinence leads to decreased basal DA levels, and whether isradipine reverses this dopaminergic effect. One hypothesis is that an isradipine-induced increase in dopamine may attenuate cocaine-seeking by alleviating the drive for cocaine, which would also increase dopamine. Our observed effect of isradipine-induced increases in DA and decreases in cocaine seeking are also consistent with a recent finding, where systemic administration of a D1/D2 receptor complex agonist decreased cocaine-reinstatement in rats [28]. Through future mechanistic studies, one could determine whether NAc specific administration of a D1/D2 receptor complex agonist is also sufficient to decrease cocaine-seeking.

LTCC antagonists and cocaine relapse-clinical implications Despite the limited number of previous investigations on LTCC modulation of cocaine relapse, one clinical study showed the ability of the LTCC inhibitor, amlodipine, to decrease craving in cocaine dependent individuals [29]—although another clinical study showed that nimodipine, given at a single dose, did not alter cue-induced cocaine craving [30]. Pre-clinically, systemic administration of fendiline, which inhibits LTCCs along with other targets, has also been shown to decrease cue and cocaineinduced reinstatement following cocaine self-administration and extinction [31]. However, the prior study did not investigate potential mechanisms nor specific brain pathways mediating fendiline's behavioral effects. Here, our findings clearly show the ability of VTA LTCC blockade to selectively attenuate cue-induced cocaine-seeking and also reveal NAC mechanisms mediating the attenuation of cocaine-seeking after systemic isradipine. In addition to our VTA findings, previous work showed that VTA isradipine administration enhances the extinction of a cocaine conditioned place preference (CPP) and also blocks cocaineprimed reinstatement of CPP [9]. In contrast to LTCC blockade, repeated VTA LTCC stimulation during cocaine CPP training enhances cocaine CPP and psychomotor sensitization, even after periods of cocaine abstinence $[5,7]$. Thus, VTA LTCCs mediate multiple behaviors associated with repeated cocaine exposure, abstinence and re-exposure to either cues or cocaine.

One key observation from our study is the emergence of the isradipine behavioral and dopaminergic effects during forced cocaine abstinence, and the lack of behavioral or neurochemical effects during cocaine taking or during forced sucrose abstinence. Previous work had shown that acute VTA LTCC blockade did not alter the expression of cocaine CPP, when CPP was tested 2 days after the last cocaine administration [9]. Our observed lack of a VTA isradipine effect on cocaine taking is similar to what has been observed with systemic isradipine administration in clinical studies, where isradipine did not alter the euphoric effects of cocaine $[32,33]$. One study did show the ability of repeated isradipine to enhance the reinforcing effects of cocaine-taking [34]. However, this enhancement may have been due to the ability of systemic LTCC blockade to decrease the aversive peripheral (i.e., cardiovascular) effects of cocaine [35-37], thereby enhancing the rating of cocaine-taking. In contrast to the lack of isradipine effects 
on cocaine taking, isradipine administration robustly attenuates cocaine-seeking and reinstatement behavior that occurs after a period of cocaine abstinence or extinction training [9]. Thus, our current findings, together with this previous work, strongly suggests that VTA LTCC blockade does not alter previously learned cocaine-taking or cocaine-seeking behavior unless there is a prolonged cocaine abstinence. These findings have important clinical implications and suggest that LTCC antagonists may have utility in preventing cue-induced relapse, in treatment-seeking cocaine dependent individuals. The data also point to regulation of DA signaling as the mechanism mediating this decrease in cocaine relapse behavior. Indeed, compounds that facilitate DA transmission and activate DA receptors have been proposed as a potential treatment option for psychostimulant addiction [26, 38, 39]. Given the critical role for VTA mechanisms in mediating behavioral responses to multiple types of drugs, an important consideration for future studies will be to determine whether VTA LTCC mechanisms also mediate cue-induced seeking for other drugs of abuse. Such investigations can provide critical, new understanding of the potential of LTCCs to serve as therapeutic targets to treat substance abuse by preventing cue-induced drug relapse.

\section{ACKNOWLEDGEMENTS}

We thank Dr. Hitoshi Morikawa for providing helpful information regarding isradipine dose, stock makeup, and solubility. We also thank Dr. Joerg Striessnig, Dr. Robert Malison, and Dr. Gustavo Angarita-Africano for helpful discussions regarding the data and the manuscript. This work was supported by National Institutes of Health (NIH) grants DA038048 (EJN, AMR and NAA), R25 GM104553 (SMH), and MH014276 (KMS).

\section{AUTHORS CONTRIBUTION}

NAA, EJN, SMH, AMR, and JLH contributed to the study design, implementation, data analysis, and interpretation of results. EJN, SMH, KMS, and SJB performed the experiments. NAA and EJN wrote the manuscript. NAA, EJN, SMH, KMS, SJB, JLH, and AMR reviewed, edited and approved the final the manuscript.

\section{ADDITIONAL INFORMATION}

Supplementary information accompanies this paper at (https://doi.org/10.1038/ s41386-018-0080-2)

Competing interest: The authors declare no conflict of interests.

Publisher's note: Springer Nature remains neutral with regard to jurisdictional claims in published maps and institutional affiliations.

\section{REFERENCES}

1. Rohsenow DJ, Niaura RS, Childress AR, Abrams DB, Monti PM. Cue reactivity in addictive behaviors: theoretical and treatment implications. Int J Addict. 1990;25 (7A-8A):957-93.

2. Sinha R. The clinical neurobiology of drug craving. Curr Opin Neurobiol. 2013;23:649-54.

3. Yahyavi-Firouz-Abadi N, See RE. Anti-relapse medications: preclinical models for drug addiction treatment. Pharmacol Ther. 2009;124:235-47.

4. Kabir ZD, Martinez-Rivera A, Rajadhyaksha AM. From Gene to Behavior: L-Type Calcium Channel Mechanisms Underlying Neuropsychiatric Symptoms. Neurotheraphy. 2017;14:588-613.

5. Martinez-Rivera A, Hao J, Tropea TF, Giordano TP, Kosovsky M, Lee A, et al. Enhancing VTA Cav1.3 L-type Ca2+channel activity promotes cocaine and mood -related behaviors via overlapping AMPA receptor mechanisms in the nucleus accumbens. Mol Psychiatry. 2017;22:1735-45.

6. Ortner NJ, Striessnig J. L-type calcium channels as drug targets in CNS disorders. Channels. 2016;10:7-13.

7. Licata SC, Freeman AY, Pierce-Bancroft AF, Pierce RC. Repeated stimulation of Ltype calcium channels in the rat ventral tegmental area mimics the initiation of behavioral sensitization to cocaine. Psychopharmacology. 2000;152:110-8.

8. Schierberl K, Hao J, Tropea TF, Ra S, Giordano TP, Xu Q, et al. Cav1.2 L-type Ca(2) $(+)$ channels mediate cocaine-induced GluA1 trafficking in the nucleus accumbens, a long-term adaptation dependent on ventral tegmental area $\mathrm{Ca}(\mathrm{v})$ 1.3 channels. J Neurosci. 2011;31:13562-75.

9. Degoulet M, Stelly CE, Ahn KC, Morikawa H. L-type Ca channel blockade with antihypertensive medication disrupts VTA synaptic plasticity and drug-associated contextual memory. Mol Psychiatry. 2015;21:394-402.

10. Phillips PE, Stuber GD, Heien ML, Wightman RM, Carelli RM. Subsecond dopamine release promotes cocaine seeking. Nature. 2003;422:614-8.

11. Solecki W, Wickham RJ, Behrens S, Wang J, Zwerling B, Mason GF, et al. Differential role of ventral tegmental area acetylcholine and $\mathrm{N}$-methyl-d-aspartate receptors in cocaine-seeking. Neuropharmacology. 2013;75C:9-18.

12. Stuber GD, Wightman RM, Carelli RM. Extinction of cocaine self-administration reveals functionally and temporally distinct dopaminergic signals in the nucleus accumbens. Neuron. 2005;46:661-9.

13. Liu Y, Dore J, Chen X. Calcium influx through L-type channels generates protein kinase $\mathrm{M}$ to induce burst firing of dopamine cells in the rat ventral tegmental area. J Biol Chem. 2007;282:8594-603.

14. Liu Y, Harding M, Pittman A, Dore J, Striessnig J, Rajadhyaksha A, et al. Cav1.2 and Cav1.3 L-type calcium channels regulate dopaminergic firing activity in the mouse ventral tegmental area. J Neurophysiol. 2014;112:1119-30.

15. Bonci A, Grillner P, Mercuri NB, Bernardi G. L-Type calcium channels mediate a slow excitatory synaptic transmission in rat midbrain dopaminergic neurons. J Neurosci. 1998;18:6693-703.

16. Venniro M, Caprioli D, Shaham Y. Animal models of drug relapse and craving: From drug priming-induced reinstatement to incubation of craving after voluntary abstinence. Prog Brain Res. 2016;224:25-52.

17. Wickham R, Solecki W, Rathbun L, Mclntosh JM, Addy NA. Ventral tegmental area alpha6beta2 nicotinic acetylcholine receptors modulate phasic dopamine release in the nucleus accumbens core. Psychopharmacology. 2013;229:73-82.

18. Hyland BI, Reynolds JN, Hay J, Perk CG, Miller R. Firing modes of midbrain dopamine cells in the freely moving rat. Neuroscience. 2002;114:475-92.

19. Koranda JL, Cone JJ, McGehee DS, Roitman MF, Beeler JA, Zhuang X. Nicotinic receptors regulate the dynamic range of dopamine release in vivo. J Neurophysiol. 2014;111:103-11.

20. Saunders BT, Yager LM, Robinson TE. Cue-evoked cocaine "craving": role of dopamine in the accumbens core. J Neurosci. 2013;33:13989-4000.

21. Reimold M, Solbach C, Noda S, Schaefer JE, Bartels M, Beneke M, et al. Occupancy of dopamine $D(1), D(2)$ and serotonin $(2 A)$ receptors in schizophrenic patients treated with flupentixol in comparison with risperidone and haloperidol. Psychopharmacology. 2007;190:241-9.

22. Rossetti ZL, Isola D, De Vry J, Fadda F. Effects of nimodipine on extracellular dopamine levels in the rat nucleus accumbens in ethanol withdrawal. Neuropharmacology. 1999;38:1361-9.

23. Rajadhyaksha A, Husson I, Satpute SS, Kuppenbender KD, Ren JQ, Guerriero RM et al. L-type $\mathrm{Ca} 2$ + channels mediate adaptation of extracellular signal-regulated kinase $1 / 2$ phosphorylation in the ventral tegmental area after chronic amphetamine treatment. J Neurosci. 2004;24:7464-76.

24. Recillas-Morales S, Sanchez-Vega L, Ochoa-Sanchez N, Caballero-Floran I, Paz-Bermudez $\mathrm{F}$, Silva I, et al. L-type $\mathrm{Ca}(2)(+)$ channel activity determines modulation of GABA release by dopamine in the substantia nigra reticulata and the globus pallidus of the rat. Neuroscience. 2014;256:292-301.

25. Parsons LH, Smith AD, Justice JB Jr.. Basal extracellular dopamine is decreased in the rat nucleus accumbens during abstinence from chronic cocaine. Synapse. 1991;9:60-5.

26. Blum K, Thanos PK, Oscar-Berman M, Febo M, Baron D, Badgaiyan RD, et al. Dopamine in the Brain: hypothesizing Surfeit or Deficit Links to Reward and Addiction. J Reward Defic Syndr. 2015;1:95-104.

27. Rossetti ZL, Hmaidan Y, Gessa GL. Marked inhibition of mesolimbic dopamine release: a common feature of ethanol, morphine, cocaine and amphetamine abstinence in rats. Eur J Pharmacol. 1992;221:227-34.

28. Hasbi A, Perreault ML, Shen MYF, Fan T, Nguyen T, Alijaniaram M, et al. Activation of Dopamine D1-D2 Receptor Complex Attenuates Cocaine Reward and Reinstatement of Cocaine-Seeking through Inhibition of DARPP-32, ERK, and DeltaFosB. Front Pharmacol. 2017;8:924.

29. Malcolm R, Brady KT, Moore J, Kajdasz D. Amlodipine treatment of cocaine dependence. J Psychoact Drugs. 1999;31:117-20.

30. Rosse RB, Alim TN, Fay-McCarthy M, Collins JP Jr., Vocci FJ Jr., Lindquist T, et al. Nimodipine pharmacotherapeutic adjuvant therapy for inpatient treatment of cocaine dependence. Clin Neuropharmacol. 1994;17:348-58.

31. Cunningham JJ, Orr E, Lothian BC, Morgen J, Brebner K. Effects of fendiline on cocaine-seeking behavior in the rat. Psychopharmacology. 2015;232:4401-10.

32. Sofuoglu M, Singha A, Kosten TR, McCance-Katz FE, Petrakis I, Oliveto A. Effects of naltrexone and isradipine, alone or in combination, on cocaine responses in humans. Pharmacol Biochem Behav. 2003;75:801-8.

33. Johnson BA, Roache JD, Ait-Daoud N, Wells LT, Mauldin JB. Effects of isradipine on cocaine-induced subjective mood. J Clin Psychopharmacol. 2004;24:180-91. 
34. Roache JD, Johnson BA, Ait-Daoud N, Mauldin JB, Thornton JE, Wells LT, et al. Effects of repeated-dose isradipine on the abuse liability of cocaine. Exp Clin Psychopharmacol. 2005;13:319-26.

35. Williams RG, Kavanagh KM, Teo KK. Pathophysiology and treatment of cocaine toxicity: implications for the heart and cardiovascular system. Can J Cardiol. 1996;12:1295-301.

36. Johnson B, Barron B, Fang B, Lamki L, Wagner L, Wells L, et al. Isradipine prevents global and regional cocaine-induced changes in brain blood flow: a preliminary study. Psychopharmacology. 1998;136:335-41.
37. Johnson BA, Wells LT, Roache JD, Wallace C, Ait-Daoud N, Wang Y. Isradipine decreases the hemodynamic response of cocaine and methamphetamine results from two human laboratory studies: results from two human laboratory studies. Am J Hypertens. 2005;18:813-22.

38. Konova AB, Moeller SJ, Goldstein RZ. Common and distinct neural targets of treatment: changing brain function in substance addiction. Neurosci Biobehav Rev. 2013;37(10 Pt 2):2806-17.

39. Shearer J. The principles of agonist pharmacotherapy for psychostimulant dependence. Drug Alcohol Rev. 2008;27:301-8. 